\title{
ECG-based Characteristics of Death or Re-infarction on the 12-Lead or 15-Lead ECG among UA/NSTEMI Patients: A Protocol for a Systematic Review and Meta-Analysis
}

Dillon J Dzikowicz (D Dillon_Dzikowicz@URMC.Rochester.edu )

University of Rochester https://orcid.org/0000-0001-7230-1927

Darcey H. Mulligan

University of Rochester

Mary G. Carey

University of Rochester

\section{Protocol}

Keywords: Electrocardiography, Acute Coronary Syndrome, Myocardial Infarction, Re-infarction, Non-ST Elevated Myocardial Infarction, Angina, Unstable, Myocardial Ischemia, Coronary Artery Disease, Risk Factors, Death

Posted Date: December 31st, 2020

DOI: https://doi.org/10.21203/rs.3.rs-33611/v2

License: (c) (1) This work is licensed under a Creative Commons Attribution 4.0 International License. Read Full License 


\section{Abstract}

\section{Background}

The incidence of unstable angina/ non-ST elevation MI (UA/NSTEMI) continues to rise, and despite treatment advancements the risks of death and re-infarction persist. The electrocardiogram (ECG) remains the first assessment of myocardial ischemia caused by UA/NSTEMI, and is performed within the first 10-minutes of emergency presentation. Once ischemia is identified on the ECG, interventions are delivered timely to decrease the risk of death and re-infarction. Since the ECG informs intervention decisions, it is crucial providers understand the prognostic value of individual characteristics on the ECG. However, characteristics on ECG are limited due to low sensitivity though newer research has aimed to overcome these limitations. This is the protocol for a systematic review and meta-analysis that will assess the significance of individual characteristics on the ECG for predicting death and re-infarction among UA/NSTEMI patients, and identify remaining gaps in clinical understanding of ECG.

\section{Methods}

This is the protocol for a systematic review and meta-analysis that will be conducted to assess the prognostic value of specific characteristics on the ECG in the emergency department for predicting reinfarction and death. Electronic databases and specific journals will be searched using predefined search terms to identify relevant studies. Eligible studies will include peer-reviewed research articles with empirical findings on the risk of re-infarction and death based on individual characteristics on the ECG. To be inclusive, we will assess characteristics on both the 12-lead and 15-lead ECG. Methodological quality of will be assessed with the Newcastle-Ottawa Quality Assessment Scale and GRADE criteria. A random effects meta-analysis and exploratory meta-regression will be conducted. Citations will be managed using EndNote X9. Meta-Essentials package for Microsoft Excel and STATA will be used to compute statistics.

\section{Discussion}

This study will systematically evaluate and quantitatively assess the evidence available on the prognostic value of individual characteristics on the ECG for the risk of death and re-infarction among UA/NSTEMI patients. By conducting this study, we will inform clinicians about the significance of characteristics on the ECG to better inform treatment decisions for UA/NSTEMI patients.

\section{Registration}

This systematic review and meta-analysis is registered with the International Prospective Register of Systematic Reviews (PROSPERO; ID CRD42020158491).

\section{Background}


More than $65 \%$ of all cases of acute coronary syndrome in the United States are diagnosed as unstable angina/ non-ST elevation myocardial infarction (UA/NSTEMI), a heterogeneous constellation of patient symptoms and diagnostic test results [1]. UA/NSTEMI is a dynamic condition causing myocardial ischemia due to a significant reduction in blood flow $[2,3]$. The most common and dangerous cause of UA/NSTEMI is due to the opening and closing of a coronary artery due to the erosion or rupture of an atherosclerotic plaque $[2,3]$. The definition for UA/NSTEMI involves a presentation with or without chest pain, but with electrocardiographic (ECG) changes consistent with ischemia (e.g. ST-segment changes) and, in NSTEMI, an elevation in cardiac biomarkers for necrosis (e.g. troponin) [2]. The majority of UA/NSTEMI patients present with chest pain and other symptoms such as arm pain and back discomfort, and normal initial cardiac biomarkers $[5,6]$. Overall, the heterogeneity in patient symptoms and diagnostic test results make decisions around timing of interventions susceptible to error despite the fact that timely interventions are needed to reduce the risk of re-infarction and death [5-8].

The risk of death and re-infarction are serious concerns among UA/NSTEMI patients, and initial treatment is based on this acute risk. Among a cohort of 11,342 UA/NSTEMI patients followed for 2-years, the reinfarction rate was UA $3.9 \%$ and NSTEMI $5.6 \%$ and the all-cause mortality rate was UA $4.9 \%$ and NSTEMI 9.5\% [9]. In a separate study analyzing two trial results which followed UA/NSTEMI patients for 30 days after hospitalization, the re-infarction rate was 2.2-4.6\% UA and 3.1\% NSTEMI and the all-cause mortality rate ranged from $0.5-0.7 \%$ for UA and $3.7 \%-7.4 \%$ for NSTEMI [10]. Despite growing emphasis on earlier interventions to prevent death and re-infarction, mortality rates have not significantly decreased $[9,11]$. This may be due to an initial underestimation of ischemic injury assessed on the ECG at presentation [68].

The ECG is crucial for informing initial treatments in UA/NSTEMI and has prognostic value for death and re-infarction, but has limitations and can be misinterpreted leading to an underestimation of ischemic injury [12]. The ECG is completed within the first 10 minutes of patient presentation per current American Heart Association/ American College of Cardiology (AHA/ACC) guidelines which enable providers to rapidly stratify patients based on greatest risk for death and re-infarction [2]. This has improved the efficiency of delivering interventions to patients who need them most. However, ECG has a number of limitations which lead to an underestimation of ischemic injury. First, some common ECG characteristics such as ST-segment depression have a low sensitivity for acute infarction [13]. This low sensitivity occurs for a number of reasons including: 1) the dynamic nature of UA/NSTEMI in which blood flow can spontaneously return or be supplied through a collateral artery; 2 ) the limited 10 -second duration of the standard ECG; and, 3 ) the lack of leads especially in the posterior wall of the myocardium making it difficult to detect ischemia $[2,13]$. Though AHA/ACC guidelines consider it reasonable to place leads over the posterior wall (V7-V9), it is not necessarily performed in clinical practice [2,14]. Lastly, the ECG is often misinterpreted by providers further leading to treatment delays $[14,15]$. Newer research has identified novel characteristics on the ECG related to UA/NSTEMI which have higher sensitivity and the potential to overcome these identified limitations $[12,16]$. Collectively, the rapidness of the ECG to assess ischemic in UA/NSTEMI is a significant advantage but a number of limitations contribute to an underestimation of ischemic injury. 
This study aims to systematically review and meta-analyze published research assessing individual characteristics on the ECG prognostic of death and re-infarction among UA/NSTEMI patients in the emergency department. The purpose for this systematic review and meta-analysis is two-fold: to inform providers about the prognostic value of characteristics on the ECG, and to identify remaining gaps in clinical understanding of ECG. In this review, we will include both common and novel characteristics on the ECG and include both 12-lead and 15-lead ECGs.

\section{Methods}

The following protocol has been written in accordance to the Guidelines for Meta-Analyses and Systematic Reviews of Observational Studies (MOOSE) and the Preferred Reporting Items for Systematic Reviews and Meta-Analyses (PRISMA-P) guidelines $[17,18]$. The PRISMA-P checklist is seen in Table 3. The protocol is registered at the International Prospective Register of Systematic Reviews (PROSPERO; ID CRD42020158491) [19].

\section{Research Question}

The PIOT (Population, Indicator, Outcome, Time) research question guiding this systematic review and meta-analysis is: Among UA/NSTEMI patients in the emergency department (Population), do specific characteristics on the ECG (Indicator) predict death and re-infarction (Outcome) in the first 28 days after initial patient presentation (Time)? No comparison component will be used. The timeframe of 28 days will be used to be consistent with the definition of re-infarction in the $4^{\text {th }}$ universal definition of myocardial infarction [20].

\section{Independent Variables}

The independent variables for this protocol are specific individual characteristics on the 12-lead or 15-lead ECG collected in the emergency department. We specify ECG in the emergency department to focus on initial emergency contact. Our expertise in this area have led us to hypothesize the following common and novel characteristics will likely be included: ST-segment depression, T-wave inversion, and QRS-T angle $[12,13,16]$. However, we will not limit our search to just these characteristics. Across different studies, measurement differences may exist. Although these characteristics have standardized measurements and corresponding normal values, individual studies may deviate from these published standards [21]. We will record details on how each characteristic is measured in this review and metaanalysis, and study the effects in a meta-regression (see below).

\section{Dependent Variable}

Re-infarction and death will be defined as the following:

Re-infarction will be defined based on the $4^{\text {th }}$ universal definition of myocardial infarction, and is defined as an acute myocardial infarction that reoccurs within 28 days of the incident myocardial infarction [20]. 
Re-infarction is diagnosed based on new ECG changes (e.g. ST-elevation, new pathological Q waves, etc.), ischemic signs and symptoms, and a $>20 \%$ increase between cardiac troponin measurements indicating myocardial necrosis [20].

Death will be defined as a composite variable as either cardiovascular death or all-cause death. Cardiovascular death will be defined according to the $10^{\text {th }}$ revision of International Classification of Disease codes I11 or I20-I25, or as death due to fatal or non-fatal myocardial infarction [22]. All-cause death will be defined as death due to any cause so forth recorded on death certificates, medical records, or national registries. Death will be a composite variable but we will perform a sub-group analysis between the two types of death if enough studies are collected.

If there is a difference in the definition in re-infarction or death in individual studies, we will record this difference as this may introduce heterogeneity. We will also record differences in follow-up whether in the same publication or across several publications, and conduct a sensitivity analysis to investigate potential bias.

\section{Moderator Variables}

Age, sex, and race are important potential moderating variables which may influence the presenting ECG $[23,24]$. Thus, we will collect information on the age, sex, and race distribution of the samples used in individual studies.

\section{Covariates}

The ECG is affected by a number of covariates which impact its interpretation and subsequently prognostic value for death and re-infarction. Such covariates include medications such as digoxin and antipsychotics, right and left bundle branch block, and left and right ventricular hypertrophy [25]. We will record these variables during data extraction as potential covariates for analysis.

\section{Participants}

The study population will be adults ( $\geq 18$ years) with UA/NSTEMI presented to the emergency department. No restrictions will be placed on participants' gender, ethnicity, or other demographic characteristics. Since the aim of the study is to determine prognosis of characteristics on the ECG and re-infarction and death, known factors influential such outcomes including age, sex, and race will be recorded and used as moderators in meta-analyses $[23,24]$.

\section{Inclusion and Exclusion Criteria}

The inclusion criteria for this study will be: 1) full-text peer-reviewed publications; 2) English language; 3 ) adult ( $\geq 18$ years) patient with emergent UA/NSTEMl; 4 ) standard, resting ECG (12-lead or 15-lead) performed in the supine position in the emergency department and 5) reporting of hazard ratio (HR), odds ratio (OR) or risk ratio (RR) and the corresponding 95\% confidence interval as the outcome measure or the 
data necessary to calculate these measures. To ensure validity, two reviewers (DJD and MGC) will independently adjudicate the diagnosis of UA/NSTEMI based on AHA/ACC guidelines [2]. We will only include papers which performed the ECG in the supine position to reduce the risk of misinterpretation related to positional effects which ultimately impact the sensitivity [26]. We will include studies that conducted serial ECG measurements which may improve the sensitivity of ECG characteristics due to a number of reasons described in the introduction [27]. Similarly, we will include papers that follow patients for more than the 28-day cutoff but focus on the first 28-days for this review. Studies reporting on overlapping samples will only be included if they reported different characteristics and/or outcomes.

We will exclude studies which enrolled participants from either inpatient hospital units, outpatient clinics, or the general population to maintain focus on emergency medicine and first medical contact. We will also exclude studies which performed ECGs greater than the standard 10-seconds such as continuous ECG monitoring because this is not the standard of care. There will be no limitation in terms of geographic region or racial background. The search period will be from the beginning of the computerization of the ECG, January $1^{\text {st }}, 1990$ to June 1,2020 [28].

For the meta-analysis portion, additional inclusion criteria will include a quality score of $\geq 5$ on the Newcastle-Ottawa Quality Assessment Scale and a moderate rating on the Grading of Recommendations, Assessment, Development and Evaluations (GRADE) criteria [29-31]. We applied this additional inclusion criteria to the meta-analysis to maintain the highest rigor in our quantitative results.

\section{Data Sources, Search Terms, and Search Strategy}

This literature review and meta-analysis will be based on systematic searches in multiple electronic literature databases, including Medline/PubMed, Web of Science, Embase, and CINAHL. Since ECG research appears in cardiovascular and emergency medicine journals, specific searches to expand capture of relevant studies will be performed using the same key words in over 20 cardiology and emergency medicine journals including: Circulation, Journal of Electrocardiology, Annals of Noninvasive Electrocardiology, European Heart Journal, The American Journal of Cardiology, and American Journal of Emergency Medicine. Lastly, we will search ClinicalTrials.gov for potential grey literature. All journals and search terms are listed in Table 1. Systematic searches will be conducted by combining every possible combination of keywords. The medical librarian, co-author DHM, will be responsible for conducting searches in the electronic databases and ClinicalTrials.gov. The principal investigator DJD will be responsible for conducting searches in cardiovascular journals with the guidance of the medical librarian. Reference lists from included articles will be checked based on keywords in the title to identify any potentially eligible studies. This in-depth and librarian guided systematic procedure ensures a comprehensive and reproducible search strategy, and the inclusion of grey literature reduces the risk of selection and detection bias [32-34]. The search results will be exported to Endnote X9 (Clarivate Analytics, PA, USA). The librarian will save the search results in an Endnote file on an encrypted, frequently backed-up computer as a historical record. 


\section{Data Extraction}

Following the search, all identified citations will be stored and assessed in EndNote X8. Next, duplicate articles will be removed. After removing all duplicates, the titles and abstracts will be screened against the inclusion and exclusion criteria. If necessary to determine inclusion, the full text will be assessed. Two reviewers (DJD and MGC) will independently assess each article to determine inclusion. Any disagreements that arise between the reviewers will be resolved through discussion and another content expert (PhD-prepared cardiac nurse) will be invited as a third reviewer to make the ultimate decision. Reasons for exclusion will be recorded and reported in the final manuscript.

After deciding which articles to include, all data entry will be completed by the two reviewers (DJD and MGC) independently for each article. The two reviewers will use a standardized data extraction form to ensure consistent data retrieval of all variables from the included studies (Table 2). The data extracted will include specific details about the publication and authorship team, study population and demographics (e.g. age, sex, race), study design methods, type of ECG and number of leads, potential ECG covariates (e.g. medications, bundle branch blocks, etc.), and critical statistical findings relevant to the purpose of this review. Importantly, we will also record information about the the measurement of specific ECG characteristics, and the determination of re-infarction and death. We will also extract information on the follow-up of patients but for the primary analysis only focus on the first 28-days. Similarly, if the study used serial ECG measurements we will extract information on each measurement but only use the most sensitive for this primary analysis. The data extraction process was piloted tested using 5 articles with an interrater reliability of $95 \%$ between the two reviewers. A third reviewer was not necessary during this pilot testing. If necessary, modifications to the standardized data extraction form will be reported in detail in the full report. If required, we will contact the authors of included studies to request missing or additional data. We will record attempts to contact authors for missing or additional data. To ensure accuracy during this process, periodic data checking and entry will be conducted.

\section{Assessment of Methodological Quality}

In this systematic review we will use two different assessments of methodological quality: the NewcastleOttawa Quality Assessment Scale and the GRADE criteria. We will use two different assessments of methodological quality because the objectives of the two assessments significantly differ but are both relevant for this systematic review. The objective of the Newcastle-Ottawa Quality Assessment Scale is to evaluate the quality of individual case-control or cohort design studies. The objective of the GRADE criteria is for the systematic appraisal of research with the goal to advise evidence-based recommendations. The aims of this systematic review and meta-analysis will be two-part: to identify remaining gaps in clinical understanding of ECG which aligns with the Newcastle-Ottawa Quality Assessment Scale and to inform providers about the prognostic value of the ECG which fit with the GRADE criteria.

The Newcastle-Ottawa Quality Assessment Scale was developed to assess the quality of non-randomized studies such as case-control and cohort studies [29]. The following characteristics will be assessed: (1) 
representativeness of the cohort; (2) selection of the non-exposed cohort; (3) ascertainment of exposure; (4) demonstration that outcome of interest was not present at the start of the study; (5) comparability of cohorts on the basis of the design or analysis; (6) assessment of outcomes; (7) follow-up period sufficiently long for outcomes to occur; and (8) adequacy of follow-up of cohorts [29]. This scale ranges from 0 to 9 indicating that studies were graded as poor to good quality, and correlates to the Agency for Healthcare Research and Quality standards [29]. The two reviewers (DJD and MGC) will score each article independently using the scale. Afterwards, the two will discuss their independent evaluations and provide a justification. If a discrepancy between quality score arises, a third independent reviewer (a PhD-prepared cardiac registered nurse) will provide a score and the average of all three reviewer scores will be used to determine study inclusion in the meta-analysis section of this study. As previously noted, inclusion of a study in the meta-analysis will require an overall score of $\geq 5$ which corresponds to good quality research [29]. Kappa (e.g. Kendall's tau) will be calculated to quantify the level of inter-rater agreement between the two or three reviewers.

GRADE is a recommended transparent framework for examining existing evidence in a systematic approach for making clinical practice recommendations $[30,31]$. The domains of GRADE include risk of bias, imprecision, inconsistency, indirectness, and publication bias [30,31]. We will use the GRADE handbook published by Cochrane to inform our evaluation of individual studies. The same protocol as described above will be followed.

\section{Meta-Analytic Power Analysis}

A random-effects meta-analytic power analysis was conducted to determine the necessary number of studies and study participants to answer the research question and achieve the specific aims of this protocol [36]. Some have argued that a minimum number of studies to be included in a meta-analysis is two [36]. Assuming a small effect size (Cohen's $d$ of 0.2), average number of participants per group ( $n=150$ participants), and high study heterogeneity, a minimum of 1,050 study participants distributed across 7 individual studies will be necessary to achieve a type I and type II error rate of $5 \%$ and $10 \%$, respectively [36]. A small effect size was assumed because individual ECG characteristics will be evaluated in this meta-analysis reducing the overall effect size. This proposal will target 12 individual studies for inclusion, yielding an estimated sample size of 1,800 subjects.

\section{Meta-Analytic Statistical Approach}

This meta-analysis will calculate relative risk as the main effect size estimate because relative risk is a cumulative and stable measure of hazard functions. Given our study has a definitive and short 28-day endpoint, a relative risk is an appropriate measure of effect size. However, to reduce the risk of bias we will include studies reporting both odds ratio and hazard ratio in this meta-analysis. We will convert odds ratio to risk ratio as described by Zhang and Yu [37]. We will perform a log transformation and perform an inverse variance weighting to combine the relative risks and hazard ratios and the corresponding standard errors. This is possible because relative risk and hazard ratio are similar measures of effect. In studies which do not report either relative risk, odds ratio, or hazard ratio, we will extract the individual cell 
data and calculate the relative risk and $95 \%$ confidence interval when possible. After converting all measures to relative risk, we will perform a log transformation to satisfy the normality assumption and weight each study by the the inverse of their variance.

Heterogeneity will be computed using Cochran $Q$ and $\mathrm{I}^{2}$ statistic. An $\mathrm{I}^{2}$ value of $\geq 50 \%$ will be considered to indicate significant heterogeneity; however, a random effects model will be used as the primary approach to due to the expected underlying methodological heterogeneity across studies $[38,29]$.

Planned sub-group analyses will be conducted to assess differences across different patient groups. Subgroup analyses will be conducted between age (mean age $<65$ years vs. mean age $\geq 65$ years), sex (mostly male $\geq 80 \%$ men vs. mostly female $\geq 80 \%$ women), and race (high racial diversity $\geq 40 \%$ non-white vs. low racial diversity $<40 \%$ non-white). These moderators were chosen because they have been associated with worse UA/NSTEMI outcomes [23,24]. Dependent on the number of studies included in this review, other potential planned sub-group analysis will include: number of ECG leads (12-lead or 15lead), final diagnosis (UA or NSTEMI), past medical history of UA/NSTEMI (yes or no), cause of death (cardiovascular death or all-cause death), time from presentation to ECG ( $\leq 10$ minutes or $>10$ minutes), and cardiac catheterization within 72 hours as recommended by AHA/ACC guidelines (yes or no) [2]. As recommended by the Cochrane handbook, a minimum number of studies to conduct these additional analyses will be 10 studies $[38,39]$.

We will also conduct sensitivity analyses to assess the impact of different decisions on the results. First, we will change the model from random effects to fixed effects so as long as $I^{2}$ value of $<50 \%$, Next, we will recalculate the relative risks by omitting one study at a time to assess for significant changes. We will assess study-level characteristics as potential sources of heterogeneity including: 1) study type (retrospective or prospective); 2 ) length of follow-up ( $\leq 28$ days or $>29$ days); 3 ) statistical measure (relative risk or hazard ratio; relative risk or odds ratio) and, 4) number of ECG leads (12-lead or 15-lead). We will also test the inclusion of less methodologically rigorous studies defined as those with a quality score of $<5$ on the Newcastle-Ottawa Quality Assessment Scale and a low/very low rating on GRADE criteria (rigorous or not vigorous) [29-31]. Publication bias will be evaluated by inspecting funnel plots, and further tested with Begg's test and Egger's test [38,39].

Lastly, we will explore the heterogeneity across studies by performing a meta-regression analysis. We will perform this analysis because of the expected heterogeneity across studies in measurement with different individual characteristics on the ECG and the use of different covariates. The analysis will determine the effects of different definitions for individual ECG characteristics, age, sex, race, length of follow-up, and listed covariates on the outcomes re-infarction and death. Each study will be weighted in the regression models using the inverse of its variance. Prior to conducting this analysis, we will ensure a minimum of 10 studies be included as recommended by Cochrane guidelines, and we will evaluate all regression-based assumptions of variable distribution, normality, and interactions $[38,39]$. 
All analyses will be performed using Meta-Essentials in Excel, and STATA 16 (STATA Software, TX) $[40,41]$. All statistical test will be two tailed, and statistical significance will be set at $P=0.05$.

\section{Discussion}

Herein, we have comprehensively described the protocol for a systematic review and meta-analysis that will evaluate and analyze characteristics on the ECG for re-infarction and death. We believe this systematic review and meta-analysis will inform providers on the prognostic significant of specific characteristics on the ECG and result in more informed clinical decision in emergency medicine. Moreover, this review will identify remaining gaps in clinical understanding of ECG and explore both common and novel characteristics on the ECG.

Recently published studies report significant findings for common (e.g. ST-segment changes) and novel (e.g. QRST angle) characteristics on the ECG prognostic of death and re-infarction with improved sensitivity $[12,13,16]$. Some characteristics of the ECG have a low sensitivity for a true infarction because the standard 10-second ECG is simply a snapshot of a very dynamic process $[12,13,16]$. Furthermore, the standard 12-lead ECG does not have posterior leads and can be easily misinterpreted $[14,15]$. Novel characteristics such as QRS-T angle on the 12-lead ECG have improved sensitivity and can overcome some of these other limitations [16]. For example, Strebel et al. (2018) reported QRS-T angle based on adjusted cutoff values based on the patient had an overall sensitivity of $69 \%$ and a specificity of $48 \%$ compared to common characteristics (e.g. ST-depression, T-wave inversion, and left bundle branch) which had an overall sensitivity of $45 \%$ and specificity of $86 \%$ [16]. Furthermore, Strebel et al. (2018) reported that QRS-T Angle predicted mortality (hazard ratio1.32 $(95 \% \mathrm{Cl}[1.26,1.40])$ per $10^{\circ}$-unit increase [16]. Thus, the inclusion of novel characteristics on the ECG may overcome the low sensitivity and be prognostic of death and re-infarction, and a comprehensive review is therefore necessary to thorough evaluate all characteristics and inform clinical practice.

To be comprehensive in this review and draw the most evidence-based conclusions, we have described a through protocol. To be inclusive, we will not limit on the type of ECG characteristics and include 12-lead and 15-lead ECG as well as serial ECG measurements. To reduce selection bias, we include articles reporting risk ratio, odds ratio, and hazard ratio. We will perform two types of quality assessment to ensure the highest rigor for each of our objectives. We will conduct planned sub-group analyses across age, sex, and race because females and minority races have poorer outcomes after UA/NSTEMI [23,24]. By addressing these key points, we make the review make comprehensive and better able to achieve its objectives.

As outlined in this protocol, this thorough review will achieve its two-fold aims: to inform providers about the prognostic value of characteristics on the ECG, and to identify remaining gaps in clinical understanding of ECG.

\section{Potential Limitations}


There are two limitations to our protocol. First, we only include full-text peer reviewed published articles and will exclude other types of scholarly works such as presentation abstracts. Although some researchers have encouraged the inclusion of other scholarly works, the inclusion of such data can introduce bias $[34,35]$. In our review we will only include peer reviewed published studies but have recruited a medical librarian to improve the robustness and quality of the search [32,33]. Furthermore, we will assess for publication bias by inspecting funnel plots and performing Begg's test and Egger's test. Secondly, our review will include risk ratio, odds ratio, and hazard ratio which may introduce methodological error. We will perform additional analyses clustering studies based on whether they reported a risk ratio, odds ratio, or hazard ratio to detect potential differences. If differences arise, we will change the analysis to a time-to-event outcome and convert all measures to hazard ratios which may be a more stable measure [42]. We will use the well-cited method proposed by Tierney et al [42].

\section{Protocol amendments}

If the present protocol is substantially amended after an initiation that may impact on the conduct of the study (including eligibility criteria, study objectives, study design, study procedures, and analysis), then this amendment will be agreed upon by all collaborators prior to the implementation and will be documented in a note to a later publication or a report under the section titled "Differences between protocol and review".

\section{Dissemination}

The results of this review will be submitted for peer-reviewed publication and will be presented at relevant cardiology conferences. The project team has commenced searching relevant studies in the relevant databases. This review is expected to be complete by June 2021 .

\section{List Of Abbreviations}

ACC: American College of Cardiology

AHA: American Heart Association

ECG: Electrocardiogram

GRADE: Grading of Recommendations, Assessment, Development and Evaluations

MOOSE: Meta-Analyses and Systematic Reviews of Observational Studies

NSTEMI: Non-ST Elevation Myocardial Infarction

PROSPERO: International Prospective Register of Systematic Reviews

PRISMA-P: Preferred Reporting Items for Systematic Reviews and Meta-Analyses 


\section{Declarations}

\section{Ethical Approval and Consent to Participate}

Not applicable.

\section{Consent for Publication}

Not applicable. Data and materials are available for reviewers upon request.

\section{Availability of Data and Materials}

The datasets created and analyzed during the current study will be available from the corresponding author upon reasonable request.

\section{Competing Interests}

The authors declare no competing interests.

\section{Funding}

DJD received the following funding for this review during his doctoral study: Sigma Theta Tau International Honor Society of Nursing Research Grant. The funder had no role in the study design; collections, analysis, or, interpretation of the data; writing of the report; or the decision to submit the findings for publication.

\section{Authors' Contributions}

DJD is the principal investigator and responsible for conceiving the review, designing search strategy, modifying data extraction tool, selecting the appropriate tool for quality appraisal, conducting the journalspecific searches, and drafting the protocol. He is the guarantor of this review, and initiated the publication of this protocol. DHM is the librarian responsible for refining the search strategy, conducting the database searches, initially designing the data extraction tool, and providing librarian support. MGC is the senior investigator who provided content expertise on the topic, study assessment and appraisal, and reviewed the methodology. All authors read, reviewed, edited, and approved the final manuscript.

\section{Acknowledgements}

Not applicable.

\section{Authors' Information}


DJD is a registered nurse and PhD candidate at the University of Rochester School of Nursing. DHM is a medical librarian at the University of Rochester Medical Center. MGC is associate professor at the University of Rochester School of Nursing, and director of clinical nursing research center at the University of Rochester Medical Center.

\section{References}

[1] Virani SS, Alonso A, Benjamin EJ, Bittencourt MS, Callaway CW, Carson AP, Chamberlain AM, Chang AR, Cheng S, Delling FN, Djousse L, Elkind MSV, Ferguson JF, Fornage M, Khan SS, Kissela BM, Knutson KL, Kwan TW, Lackland DT, Lewis TT, Lichtman JH, Longenecker CT, Loop MS, Lutsey PL, Martin SS, Matsushita K, Moran AE, Mussolino ME, Perak AM, Rosamond WD, Roth GA, Sampson UKA, Satou GM, Schroeder EB, Shah SH, Shay CM, Spartano NL, Stokes A, Tirschwell DL, VanWagner LB, Tsao CW; American Heart Association Council on Epidemiology and Prevention Statistics Committee and Stroke Statistics Subcommittee. Heart Disease and Stroke Statistics-2020 Update: A Report From the American Heart Association. Circulation. 2020;141(9):e139-e596. PMID: 31992061.

[2] Amsterdam EA, Wenger NK, Brindis RG, Casey DE, Ganiats TG, Holmes DR, Jaffe AS, Jneid H, Kelly RF, Kontos MC, Levine GN. 2014 AHA/ACC guideline for the management of patients with non-ST-elevation acute coronary syndromes: a report of the American College of Cardiology/American Heart Association Task Force on Practice Guidelines. Journal of the American College of Cardiology. 2014; 64(24):e139-228.

[3] Gambhir DS. First $24 \mathrm{~h}$ in the management of non-ST segment elevation myocardial infarction. European Heart Journal Supplements. 2018; 20(suppl_B):B29-38.

[4] Ängerud KH, Sederholm Lawesson S, Isaksson RM, Thylén I, Swahn E. Differences in symptoms, first medical contact and pre-hospital delay times between patients with ST-and non-ST-elevation myocardial infarction. European Heart Journal: Acute Cardiovascular Care. 2019;8(3):201-7.

[5] Giannitsis E, Biener M, Hund H, Mueller-Hennessen M, Vafaie M, Gandowitz J, Riedle C, Löhr J, Katus HA, Stoyanov KM. Management and outcomes of patients with unstable angina with undetectable, normal, or intermediate hsTnT levels. Clinical Research in Cardiology. 2019 19:1-2.

[6] Corcoran D, Grant P, Berry C. Risk stratification in non-ST elevation acute coronary syndromes: Risk scores, biomarkers and clinical judgment. IJC Heart \& Vasculature. 2015 1;8:131-7.

[7] Engel J, Heeren MJ, van der Wulp I, de Bruijne MC, Wagner C. Understanding factors that influence the use of risk scoring instruments in the management of patients with unstable angina or non-ST-elevation myocardial infarction in the Netherlands: a qualitative study of health care practitioners' perceptions. BMC health services research. 2014; 14(1):418.

[8] Engel J, Poldervaart JM, Van Der Wulp I, Reitsma JB, De Bruijne MC, Bunge JJ, Cramer MJ, Tietge WJ, Uijlings R, Wagner $\mathrm{C}$. Selecting patients with non-ST-elevation acute coronary syndrome for coronary angiography: a nationwide clinical vignette study in the Netherlands. BMJ open. 2017; 7(1):e011213. 
[9] Alnasser SM, Huang W, Gore JM, Steg PG, Eagle KA, Anderson Jr FA, Fox KA, Gurfinkel E, Brieger D, Klein W, Van de Werf F. Late consequences of acute coronary syndromes: global registry of acute coronary events (GRACE) follow-up. The American journal of medicine. 2015 ;128(7):766-75.

[10] Puelacher C, Gugala M, Adamson PD, Shah A, Chapman AR, Anand A, Sabti Z, Boeddinghaus J, Nestelberger T, Twerenbold R, Wildi K. Incidence and outcomes of unstable angina compared with nonST-elevation myocardial infarction. Heart. 2019;105(18):1423-31.

[11] Li Y, Zhang Z, Xiong X, Cho WC, Hu D, Gao Y, Shang H, Xing Y. Immediate/early vs. Delayed invasive strategy for patients with non-ST-segment elevation acute coronary syndromes: a systematic review and Meta-Analysis. Frontiers in physiology. 2017; 8:952.

[12] Chen PF, Tang L, Pei JY, Yi JL, Xing ZH, Fang ZF, Zhou SH, Hu XQ. Prognostic value of admission electrocardiographic findings in non-ST-segment elevation myocardial infarction. Clinical Cardiology. 2020 43(6):574-580.

[13] Wiśniewski P, Rostoff P, Gajos G, Nessler J, Kruszelnicka O. Predictive value of electrocardiographic ST-segment elevation myocardial infarction equivalents for detecting acute coronary artery occlusion in patients with non-ST-segment elevation myocardial infarction. Kardiol Pol. $2019 ; 77(6): 624-631$.

[14] Khan JN, Chauhan A, Mozdiak E, Khan JM, Varma C. Posterior myocardial infarction: are we failing to diagnose this? Emerg Med J. 2012;29(1):15-8.

[15] Faramand Z, Frisch SO, DeSantis A, Alrawashdeh M, Martin-Gill C, Callaway C, Al-Zaiti S. Lack of Significant Coronary History and ECG Misinterpretation Are the Strongest Predictors of Undertriage in Prehospital Chest Pain. J Emerg Nurs. 2019;45(2):161-168.

[16] Strebel I, Twerenbold R, Wussler D, Boeddinghaus J, Nestelberger T, du Fay de Lavallaz J, Abächerli R, Maechler P, Mannhart D, Kozhuharov N, Rubini Giménez M, Wildi K, Sazgary L, Sabti Z, Puelacher C, Badertscher P, Keller DI, Miró Ò, Fuenzalida C, Calderón S, Martin-Sanchez FJ, Iglesias SL, Osswald S, Mueller C, Reichlin T. Incremental diagnostic and prognostic value of the QRS-T angle, a 12-lead ECG marker quantifying heterogeneity of depolarization and repolarization, in patients with suspected non-STelevation myocardial infarction. Int J Cardiol. 2019;277:8-15.

[17] Stroup DF, Berlin JA, Morton SC, Olkin I, Williamson GD, Rennie D, Moher D, Becker BJ, Sipe TA, Thacker SB. Meta-analysis of observational studies in epidemiology: a proposal for reporting. JAMA. 2000; 283(15):2008-12.

[18] Moher D, Shamseer L, Clarke M, Ghersi D, Liberati A, Petticrew M, Shekelle P, Stewart LA. Preferred reporting items for systematic review and meta-analysis protocols (PRISMA-P) 2015 statement. Systematic reviews. 2015;4(1):1. 
[19] Booth A, Clarke M, Dooley G, Ghersi D, Moher D, Petticrew M, Stewart L. The nuts and bolts of PROSPERO: an international prospective register of systematic reviews. Systematic reviews. 2012;1(1):2.

[20] Thygesen K, Alpert JS, Jaffe AS, Chaitman BR, Bax JJ, Morrow DA, White HD. Fourth universal definition of myocardial infarction (2018). Journal of the American College of Cardiology. 2018;72(18):2231-64.

[21] Kligfield P, Gettes LS, Bailey JJ, Childers R, Deal BJ, Hancock EW, Van Herpen G, Kors JA, Macfarlane $P$, Mirvis DM, Pahlm O. Recommendations for the standardization and interpretation of the electrocardiogram: part I: the electrocardiogram and its technology a scientific statement from the American Heart Association Electrocardiography and Arrhythmias Committee, Council on Clinical Cardiology; the American College of Cardiology Foundation; and the Heart Rhythm Society endorsed by the International Society for Computerized Electrocardiology. Journal of the American College of Cardiology. 2007 Mar 13;49(10):1109-27.

[22] World Health Organization. The ICD-10 classification of mental and behavioural disorders: diagnostic criteria for research. World Health Organization; 1993.

[23] Mefford MT, Li BH, Qian L, Reading SR, Harrison TN, Scott RD, Cavendish JJ, Jacobsen SJ, Kanter MH, Woodward M, Reynolds K. Sex-Specific Trends in Acute Myocardial Infarction Within an Integrated Healthcare Network, 2000 Through 2014. Circulation. 2020;141(7):509-19.

[24] Chi GC, Kanter MH, Li BH, Qian L, Reading SR, Harrison TN, Jacobsen SJ, Scott RD, Cavendish JJ, Lawrence JM, Tartof SY. Trends in Acute Myocardial Infarction by Race and Ethnicity. Journal of the American Heart Association. 2020;9(5):e013542.

[25] Pollak AW. Confounders of ST-Elevation Myocardial Infarction. Electrocardiogram in Clinical Medicine. 2020:69-74.

[26] Adams MG, Drew BJ. Body position effects on the ECG: implication for ischemia monitoring. Journal of Electrocardiology. 1997; 30(4):285-91.

[27] Lehmacher J, Neumann JT, Sörensen NA, Goßling A, Haller PM, Hartikainen TS, Clemmensen P, Zeller T, Blankenberg S, Westermann D. Predictive Value of Serial ECGs in Patients with Suspected Myocardial Infarction. Journal of clinical medicine. 2020;9(7):2303.

[28] Smulyan H. The computerized ECG: friend and foe. The American journal of medicine. 2019; 132(2):153-60.

[29] Wells GA. The Newcastle-Ottawa Scale (NOS) for assessing the quality of non andomized studies in meta-analyses. http://www. Ohri. Ca/programs/clinical_epidemiology/oxford. Asp. 2001.

[30] Guyatt G, Oxman AD, Akl EA, Kunz R, Vist G, Brozek J, Norris S, Falck-Ytter Y, Glasziou P, Debeer H, Jaeschke R. GRADE guidelines: 1. Introduction-GRADE evidence profiles and summary of findings 
tables. Journal of clinical epidemiology. 2011;64(4):383-94.

[31] Balshem H, Helfand M, Schünemann HJ, Oxman AD, Kunz R, Brozek J, Vist GE, Falck-Ytter Y, Meerpohl J, Norris S, Guyatt GH. GRADE guidelines: 3. Rating the quality of evidence. Journal of clinical epidemiology. 2011;64(4):401-6.

[32] Townsend WA, Anderson PF, Ginier EC, MacEachern MP, Saylor KM, Shipman BL, Smith JE. A competency framework for librarians involved in systematic reviews. Journal of the Medical Library Association: JMLA. 2017;105(3):268.

[33] Spencer AJ, Eldredge JD. Roles for librarians in systematic reviews: a scoping review. Journal of the Medical Library Association: JMLA. 2018; 106(1):46.

[34] Schmucker CM, Blümle A, Schell LK, Schwarzer G, Oeller P, Cabrera L, von Elm E, Briel M, Meerpohl JJ. Systematic review finds that study data not published in full text articles have unclear impact on metaanalyses results in medical research. PloS One. 2017; 12(4):e0176210.

[35] Hartling L, Featherstone R, Nuspl M, Shave K, Dryden DM, Vandermeer B. Grey literature in systematic reviews: a cross-sectional study of the contribution of non-English reports, unpublished studies and dissertations to the results of meta-analyses in child-relevant reviews. BMC medical research methodology. 2017;17(1):64.

[36] Valentine JC, Pigott TD, Rothstein HR. How many studies do you need? A primer on statistical power for meta-analysis. Journal of Educational and Behavioral Statistics. 2010; 35(2):215-47.

[37] Zhang J, Kai FY. What's the relative risk? A method of correcting the odds ratio in cohort studies of common outcomes. JAMA. 1998; 280(19):1690-1.

[38] Macaskill P, Gatsonis C, Deeks J, Harbord R, Takwoingi Y. Cochrane handbook for systematic reviews of diagnostic test accuracy. Version 0.9. 0. London: The Cochrane Collaboration. 2010.

[39] Higgins JP, Thomas J, Chandler J, Cumpston M, Li T, Page MJ, Welch VA, editors. Cochrane handbook for systematic reviews of interventions. John Wiley \& Sons; 2019.

[40] Suurmond R, van Rhee H, Hak T. Introduction, comparison, and validation of Meta-Essentials: A free and simple tool for meta-analysis. Research synthesis methods. 2017; 8(4):537-53.

[41] Wallace BC, Schmid CH, Lau J, Trikalinos TA. Meta-Analyst: software for meta-analysis of binary, continuous and diagnostic data. BMC medical research methodology. 2009; 9(1):80.

[42]. Tierney JF, Stewart LA, Ghersi D, Burdett S, Sydes MR. Practical methods for incorporating summary time-to-event data into meta-analysis. Trials. 2007;8:16.

\section{Tables}


Table 1. Search Strategy for Protocol for ECG-based Characteristics of Death or Reinfarction on the 12-Lead or 15-Lead ECG among UA/NSTEMI Patients. 

“cardiovascular nursing” [tiab]) AND (“Myocardial Infarction” [Mesh] OR “Non-ST Elevated Myocardial Infarction” [Mesh] OR "Myocardial Ischemia” [Mesh] OR “Myocardial Reperfusion” [Mesh] OR “Myocardial Revascularization” [Mesh] OR “Ischemic Preconditioning” [Mesh] OR “Acute Coronary Syndrome” [Mesh] OR “Angina, Unstable” [Mesh] OR "Angina Pectoris" [Mesh] OR “Coronary Artery Disease" [Mesh] OR "Coronary Vessels" [Mesh] OR "Coronary Circulation" [Mesh] OR "myocardial infarction" [tiab] OR "Non-ST elevated myocardial infarction" [tiab] OR "myocardial ischemia” [tiab] OR "myocardial reperfusion" [tiab] OR "reinfarction" [tiab] OR "re-infarction" [tiab] OR “myocardial revascularization" [tiab] OR “ischemic pre-conditioning” [tiab] OR “acute coron ary syndrome" [tiab] OR "unstable angina” [tiab] OR "chest pain" [tiab] OR "chest pains" [tiab] OR "angina pectoris" [tiab] OR "atherosclerosis" [tiab] OR "coronary artery dis ease" [tiab] OR "coronary arteries" [tiab] OR "coronary artery" [tiab] OR "coronary circulation” [tiab] OR “UA/NSTEMI”[tiab]) AND (“12 lead ECG” [tiab] OR “12 lead electrocardiogram” [tiab] OR “15 lead ECG”[tiab] OR “15 lead electrocardiogram” [tiab] OR “Electrocardiography” [Mesh] OR “Electrocardiography” [tiab] OR “ECG” [tiab] OR “EKG” [tiab] OR "electrocardiogram” [tiab] OR “electrocardiography” [tiab] OR “diagn os tic tests, routine” [MeSH Terms] OR “diagnostic measure” [tiab] OR “diagnostic procedure” [tiab] OR “diagnostic procedures” [tiab] OR “diagnostic test” [tiab] OR “diagnostic tests" [tiab] OR “diagnostic techniques"[tiab]))) AND (“Death”[Mesh] OR “Fatal Outcome” [Mesh] OR “Cause of Death” [Mesh] OR “Mortality” [Mesh] OR “dead” [tiab] OR “death" [tiab] OR "fatality” [tiab] OR "fatal outcome" [tiab]) AND ("Sensitivity and Specificity" [Mesh] OR "Prognosis" [Mesh] OR "risk ratio" [tiab] OR "odds ratio" [tiab] OR “risk" [tiab] OR "hazard ratio" [tiab] OR "hazard” [tiab] OR "probability”[tiab] OR “harm reduction” [tiab] OR "populations at risk” [tiab] OR "population at risk" [tiab]))

\begin{tabular}{l|l} 
& \\
\hline ience & TS \\
& TS
\end{tabular}

TS= ("myocardial infarction" OR "Non-ST elevated Myocardial in farction" OR “myocardial ischemia" OR "acute coronary syndrome" OR "unstable an gina" OR “ Angina Pectoris" OR "coronary artery disease" OR "coronary vessels" OR "coronary circulation" OR " atherosclerosis" OR "chest pain*" OR "Coronary artery" OR "UA/NSTEMI" OR "re-infarction" OR "reinfarction")

TS= (“12 lead ECG” OR “15 lead ECG” OR “12 lead electrocardiogram” OR “15 lead electrocardiogram” OR “twelve lead ECG” OR "fifteen lead ECG” OR “twelve lead electrocardiogram" OR “fifteen electrocardiogram” OR "electrocardiogram” OR "electrocardiography" OR “ECG” OR "EKG” OR "diagnostic test*” OR “diagn ostic measure*” OR "diagnostic procedure*" OR "diagnos tic techniques")

TS=( "death" OR "death" OR "mortality" OR "cause of death" OR "fatal outcome" OR "died" OR "die" OR "fatality" OR "fatal")

TS=("sensitivity and specificity" OR "prognosis" OR "risk ratio" OR "risk" OR "odds ratio" OR "hazard ratio" OR "hazard" "probability" OR "harm reduction" OR "population* at risk")

(\#5 AND \#4 AND \#3 AND \#2 AND \# 1)

('emergency service, hos pital'/exp OR 'emergency service, hos pital' OR 'emergency department,':ti,ab OR 'emergency departments':ti,ab OR 'emergency nursing':ti,ab OR 'emergency medicine':ti,ab OR triage:ti,ab OR 'cardiovascular nursing':ti,ab OR 'coronary care nursing':ti,ab) AND ('myocardial infarction':ti,ab OR 'non st segment elevation myocardial infarction':ti,ab OR 'myocardial ischemia':ti,ab OR 'acute coronary syndrome':ti,ab OR 'u nstable angina':ti,ab OR 'chest pain s':ti,ab OR 'an gina pectoris':ti,ab OR 'atherosclerosis':ti,ab OR 'coronary artery disease':ti,ab OR 'coronary arteries':ti,ab OR 'coronary circulation':ti, ab OR 'ua/nstemi':ti,ab OR 'heart infarction'/exp OR 'non st segment elevation myocardial infarction'/exp OR 'heart muscle ischemia'/exp OR heart muscle reperfusion'/exp OR 're-infarction'/exp OR 'reinfarction'/ecp OR 'heart muscle revascularization'/exp OR 'ischemic preconditioning'/exp OR 'acute coronary syndrome'/exp OR 'unstable angina pectoris'/exp OR 'an gina pectoris'/exp OR 'coronary artery disease'/exp OR 'coronary blood vessel'/exp) AND ('12 lead ecg':ti,ab OR '15 lead ecg':ti.ab OR 'twelve lead electrocardiogram':ti,ab OR 'fifteen lead electrocardiogram':ti.ab OR 'twelve lead ecg':ti,ab OR 'fifteen lead ecg':ti.ab OR 'ecg':ti,ab OR 'ekg':ti,ab OR ‘diagnos tic test':ti,ab OR 'diagnostic procedure':ti,ab OR 'diagnostic measure':ti,ab OR ‘diagnostic test'/exp OR '12 lead electrocardiogram'/exp OR 'electrocardiogram'/exp OR 'electrocardiography'/exp) AND ('death'/exp OR 'fatality'/exp OR 'cause of death'/exp OR 'fatal outcome':ti,ab OR 'morality':ti,ab OR 'death':ti,ab OR 'dead':ti,ab) AND ('sensitivity and specificity'/exp OR 'prognosis'/exp OR 'risk ratio'/exp OR 'odds ratio'/exp OR 'risk'/exp OR 'hazard ratio'/exp OR 'hazard'/exp OR 'probability'/exp OR 'harm redu ction'/exp OR 'population at risk'/exp)

1 ((MH “Emergency Service+") OR (MH “Emergency Medical Services+”) OR “emergency services” OR (MH “Cardiovascular Nursing+”) OR “cardiovascular nurse” OR (MH “Triage”) OR (MH “Coronary Care Nursing”) ) AND 2 (MH “Myocardial Infarction+") OR (MH “Myocardial Ischemia+") OR (MH “Myocardial Reperfusion”) OR (MH "Myocardial Revascularization+") OR (MH "Ischemic Preconditioning, Myocardial”) OR (MH "Angina, Unstable”) OR (MH "Angina Pectoris+") OR (MH “Acute Coronary Syndrome") OR "non-st- segment-elevation acute coronary syndrome" OR (MH "Coronary Arteriosclerosis") OR AB "chest pains" OR "atherosclerosis" OR "coronary arteries" OR "coronary circulation" OR "heart infarction" OR 're-infarction" OR "reinfarction" OR heart muscle ischemia” OR "heart muscle revascularization" OR "unstable Angina Pectoris" OR "coronary blood vessel" AND 3 (MH "Electrocardiography+") OR AB "electrocardiogram" OR "ecg” OR "ekg" OR "12 lead electrocardiogram" OR "15 lead electrocardiogram" OR "12 lead ecg" OR "15 lead ecg” OR "twelve lead ecg” OR "fifteen lead ecg" OR "twelve lead electrocardiogram" OR "fifteen lead electrocardiogram" AND 4 (MH "Death+") OR (MH "Fatal Outcome") OR (MH "Cause of Death") OR (MH "Mortality+") AND 5 (MH "Sensitivity and Specificity") OR (MH "Prognosis+") OR (MH “Odds Ratio") OR (MH "Harm Reduction") OR AB “" risk ratio" OR "risk" OR "hazard ratio" OR "hazard" OR "probability" OR "population at risk" 1 AND 2 AND 3 AND 4 AND 5

\begin{tabular}{|c|c|}
\hline SA Jou mals & $\begin{array}{l}\text { “ECG OR EKG OR Electrocardiogram" AND "Non-ST Elevation Myocardial Infarction OR Unstable Angina" AND "Risk Stratification OR Risk Management" AND ["Patient } \\
\text { Outcomes" OR "Death" OR "Mortality" OR "Re-infarction" OR "Reinfarction"] }\end{array}$ \\
\hline thm & $\begin{array}{l}\text { Electrocardiogram OR ECG OR EKG AND Non-ST Elevation Myocardial Infarction OR NSTEMI OR Unstable Angina OR UA AND Death OR Mortality OR Re-infarction OR } \\
\text { Reinfarction }\end{array}$ \\
\hline
\end{tabular}

(ECG OR EKG OR Electrocardiogram) AND (Non-ST Elevation Myocardial Infarction OR Unstable Angina) AND (Risk Stratification OR Risk Management) AND (Patient rdiography/ $\quad$ Outcomes OR Death OR Mortality OR Re-infarction OR Reinfarction) AND (Odds Ratio OR Risk Ratio OR Hazard Ratio)

Page $18 / 24$ 


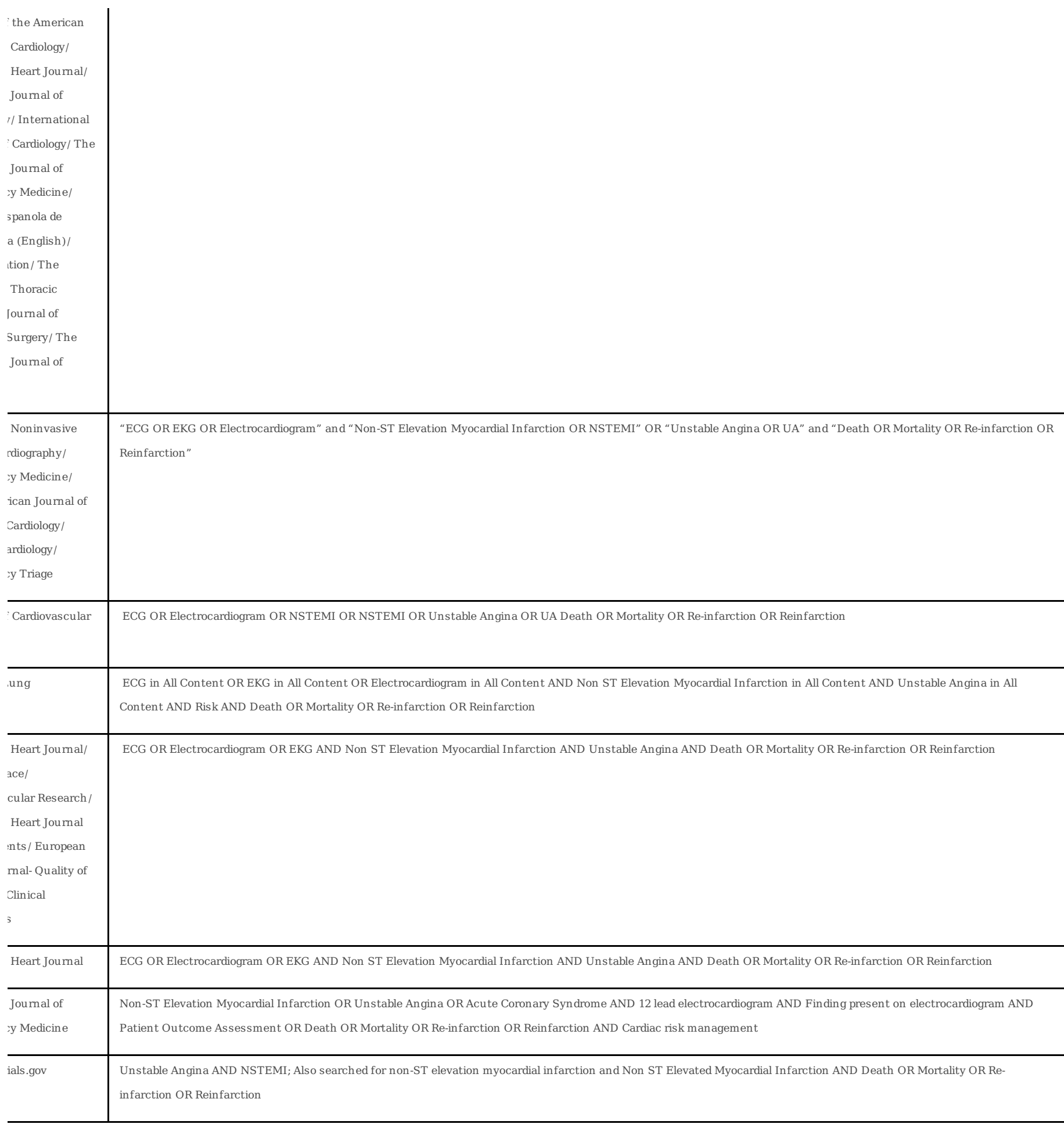

Table 2. Data Extraction Form for Protocol for ECG-based Predictors of Re-infarction or Death on the Hospital Admission 12-Lead ECG among UA/NSTEMI Patients. 


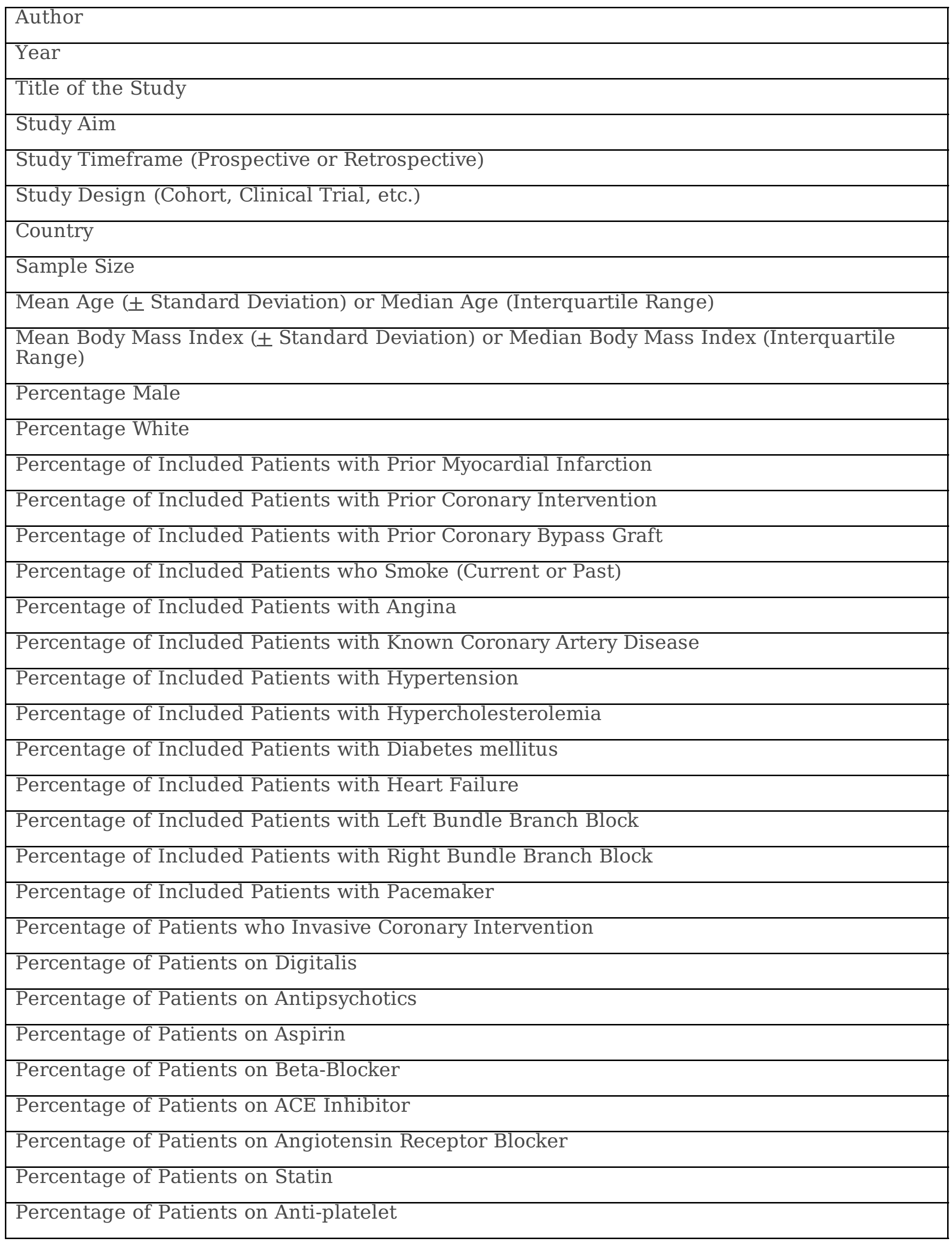




\begin{tabular}{|l|}
\hline Percentage of Patients on Anti-coagulant \\
\hline Type of ECG Recording (12-lead or 15-lead) \\
\hline Time from Presentation to Emergency Department and Invasive Coronary Intervention \\
\hline Time from Presentation to ECG Recording \\
\hline ECG Characteristics of Interest (i.e. ST-Segment Depression, T Wave Inversion, etc.) \\
\hline Definition of ECG Characteristics of Interest \\
\hline Primary Endpoint \\
\hline Definition of Primary Endpoint \\
\hline Associated Statistic (Relative Risk, Odds Ratio, Hazard Ratio) \\
\hline Associated Statistic 95\% Confidence Interval \\
\hline Sensitivity \\
\hline Specificity \\
\hline Negative Predictive Value \\
\hline Positive Predictive Value \\
\hline Length of Follow-Up \\
\hline Covariates Adjusted \\
\hline Key Conclusions \\
\hline Comments \\
\hline
\end{tabular}

Table 3. PRISMA-P 2015 Checklist for Electrocardiogram-based Predictors of Re-infarction or Death on the Admission 12-Lead and 15-Lead ECG among UA/NSTEMI Patients: A Systematic Review and Meta-Analysis Protocol 


\section{Yes No}

\section{ADMINISTRATIVE INFORMATION}

Title

\begin{tabular}{ccccc}
\hline Identification & 1a $\begin{array}{l}\text { Identify the report as a protocol of a systematic } \\
\text { review }\end{array}$ & $\mathrm{X}$ & $\square \begin{array}{l}11,18, \\
119,121\end{array}$ \\
\hline Update & 1b $\begin{array}{l}\text { If the protocol is for an update of a previous } \\
\text { systematic review, identify as such }\end{array}$ & $\square$ & $\mathrm{X}$ & NA \\
\hline
\end{tabular}

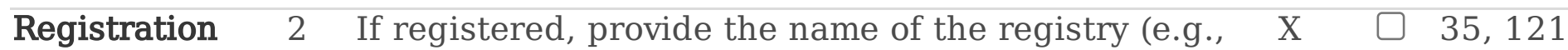
PROSPERO) and registration number in the Abstract

\section{Authors}

Contact

3a Provide name, institutional affiliation, and e-mail address of all protocol authors; provide physical $\mathrm{X} \square \quad \square-21$ mailing address of corresponding author

3b Describe contributions of protocol authors and $\mathrm{X}$ 427-435

Contributions identify the guarantor of the review

\begin{tabular}{lllll}
\hline Amendments $\quad 4 \quad \begin{array}{l}\text { If the protocol represents an amendment of a } \\
\text { previously completed or published protocol, identify } \\
\text { as such and list changes; otherwise, state plan for } \\
\text { documenting important protocol amendments }\end{array}$ & $\begin{array}{l}\mathrm{X} \\
\text { NA }\end{array}$
\end{tabular}

\section{Support}

Sources

5a Indicate sources of financial or other support for $\mathrm{X}$

$422-426$ the review

Sponsor

$5 b$

$$
\text { Provide name }
$$

Role of

$5 c$

Describe roles of funder(s), sponsor(s), and/or institution(s), if any, in developing the protocol

sponsor/funder

\section{INTRODUCTION}

\begin{tabular}{llllll}
\hline Rationale & 6 & $\begin{array}{l}\text { Describe the rationale for the review in the context } \\
\text { of what is already known }\end{array}$ & $X$ & $\square$ & $106-117$ \\
Objectives & 7 & $\begin{array}{l}\text { Provide an explicit statement of the question(s) the } \\
\text { review will address with reference to participants, } \\
\text { interventions, comparators, and outcomes (PICO) }\end{array}$ & & & \\
\hline & &
\end{tabular}

\section{METHODS}




\begin{tabular}{lllll}
\hline $\begin{array}{l}\text { Eligibility } \\
\text { criteria }\end{array}$ & $8 \begin{array}{l}\text { Specify the study characteristics (e.g., PICO, study } \\
\text { design, setting, time frame) and report } \\
\text { characteristics (e.g., years considered, language, } \\
\text { publication status) to be used as criteria for } \\
\text { eligibility for the review }\end{array}$ & &
\end{tabular}

\begin{tabular}{|c|c|c|c|}
\hline $\begin{array}{l}\text { Information } \\
\text { sources }\end{array}$ & 9 & $\begin{array}{l}\text { Describe all intended information sources (e.g., } \\
\text { electronic databases, contact with study authors, } \\
\text { trial registers, or other grey literature sources) with } \\
\text { planned dates of coverage }\end{array}$ & $\mathrm{X}$ \\
\hline
\end{tabular}

$\begin{array}{lll}\begin{array}{l}\text { Search } \\ \text { strategy }\end{array} & 10 \begin{array}{l}\text { Present draft of search strategy to be used for at } \\ \text { least one electronic database, including planned } \\ \text { limits, such that it could be repeated }\end{array} & \mathrm{X} \quad \square \text { Table 1 }\end{array}$

\section{STUDY RECORDS}

\begin{tabular}{|c|c|c|c|c|}
\hline $\begin{array}{l}\text { Data } \\
\text { management }\end{array}$ & $\begin{array}{l}\text { 11a Describe the mechanism(s) that will be used to } \\
\text { manage records and data throughout the review }\end{array}$ & $\mathrm{X}$ & $\square$ & $219-221$ \\
\hline $\begin{array}{l}\text { Selection } \\
\text { process }\end{array}$ & $\begin{array}{l}\text { 11b State the process that will be used for selecting } \\
\text { studies (e.g., two independent reviewers) through } \\
\text { each phase of the review (i.e., screening, eligibility, } \\
\text { and inclusion in meta-analysis) }\end{array}$ & $\mathrm{X}$ & $\square$ & $223-230$ \\
\hline $\begin{array}{l}\text { Data } \\
\text { collection } \\
\text { process }\end{array}$ & $\begin{array}{l}\text { 11c Describe planned method of extracting data from } \\
\text { reports (e.g., piloting forms, done independently, in } \\
\text { duplicate), any processes for obtaining and } \\
\text { confirming data from investigators }\end{array}$ & $\mathrm{X}$ & $\square$ & $231-248$ \\
\hline Data items & $\begin{array}{l}12 \text { List and define all variables for which data will be } \\
\text { sought (e.g., PICO items, funding sources), any pre- } \\
\text { planned data assumptions and simplifications }\end{array}$ & $\mathrm{X}$ & $\square$ & $\begin{array}{l}\text { 131-141; } \\
\text { 160-169; } \\
\text { Table 2 }\end{array}$ \\
\hline $\begin{array}{l}\text { Outcomes and } \\
\text { prioritization }\end{array}$ & $\begin{array}{l}\text { List and define all outcomes for which data will be } \\
\text { sought, including prioritization of main and } \\
\text { additional outcomes, with rationale }\end{array}$ & $\mathrm{X}$ & $\square$ & $142-159$ \\
\hline $\begin{array}{l}\text { Risk of bias in } \\
\text { individual } \\
\text { studies }\end{array}$ & $\begin{array}{l}\text { Describe anticipated methods for assessing risk of } \\
\text { bias of individual studies, including whether this } \\
\text { will be done at the outcome or study level, or both; } \\
\text { state how this information will be used in data } \\
\text { synthesis }\end{array}$ & $\mathrm{X}$ & $\square$ & $249-280$ \\
\hline
\end{tabular}

DATA

Synthesis

15a Describe criteria under which study data will be

$\mathrm{X}$

292-343

quantitatively synthesized 
$15 \mathrm{~b}$ If data are appropriate for quantitative synthesis, X describe planned summary measures, methods of 274-275; handling data, and methods of combining data from 292-308 studies, including any planned exploration of consistency (e.g., $I^{2}$, Kendall's tau)

15c Describe any proposed additional analyses (e.g., $\mathrm{X}$ $307-324$ sensitivity or subgroup analyses, meta-regression)

$15 \mathrm{~d}$ If quantitative synthesis is not appropriate, $\mathrm{X} \quad \mathrm{NA}$ describe the type of summary planned

Meta-bias(es) $16 \quad$ Specify any planned assessment of meta-bias(es) X (e.g., publication bias across studies, selective reporting within studies)

Confidence in cumulative evidence 